\title{
Inflammatory potential of the diet and risk of sarcopenia and its components
}

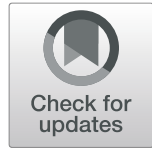

\author{
Amir Bagheri ${ }^{1,2}$, Sanaz Soltani ${ }^{1,2}$, Rezvan Hashemi ${ }^{3^{*}}$, Ramin Heshmat ${ }^{4}$, Ahmadreza Dorosty Motlagh $^{2}$ and
} Ahmad Esmaillzadeh $2,5,6^{*}$

\begin{abstract}
Background: Despite a large body of evidence on the link between dietary inflammatory index (DII) and several chronic conditions, limited data are available about the association of DII and sarcopenia. This study aimed to examine the relationship between inflammatory potential of the diet (as measured by DII) and sarcopenia and its components among community-dwelling elderly population.
\end{abstract}

Methods: This population-based cross-sectional study was performed in 2011 among 300 elderly people (150 men and 150 women) aged $\geq 55$ years, who were selected using cluster random sampling method. Dietary assessment was done using a pre-tested food frequency questionnaire. Energy-adjusted DII was calculated based on earlier studies. Sarcopenia and its components were determined based on the European Working Group on Sarcopenia (EWGSOP) definition.

Results: Mean age of study participants was $66.7 \pm 7.7 \mathrm{y}$. Subjects in the highest tertile of DII score (i.e. those with a more pro-inflammatory diet) were more likely to be older $(P=0.02)$. The prevalence of sarcopenia $(P=0.016)$ and low muscle mass $(P=0.041)$ was significantly higher among subjects in the top tertile compared with those in the bottom tertile of DII. After adjustment for potential confounders, those with the highest DII were 2.18 times (95\% Cl: 1.01-4.74) more likely to have sarcopenia than those with the lowest DII. With regard to components of sarcopenia, subjects in the top tertile of DII had not significantly greater odds of low muscle mass (OR: 1.38; 95\% Cl: 0.72-2.63), abnormal handgrip strength (OR: 0.97; 95\% Cl: 0.49-1.89), and abnormal gait speed (OR: 1.61; 95\% Cl: 0.84-3.08) than those in the bottom tertile.

Conclusions: In conclusion, a diet with more pro-inflammatory potential was associated with a greater odds of sarcopenia. Further studies are required to confirm these findings.

Keywords: Dietary inflammatory index, Sarcopenia, Muscle mass, Muscle strength

\section{Introduction}

Sarcopenia is described as a geriatric syndrome determined by wasting muscle mass in addition to reduced muscle strength and/or physical performance [1].

\footnotetext{
* Correspondence: r_hashemi@tums.ac.ir; a-esmaillzadeh@tums.ac.ir

${ }^{3}$ Department of Geriatric Medicine, Ziaeian Hospital, Tehran University of Medical Sciences, Tehran, Iran

${ }^{2}$ Department of Community Nutrition, School of Nutritional Sciences and Dietetics, Tehran University of Medical Sciences, P.O. Box 14155-6117, Tehran, Iran

Full list of author information is available at the end of the article
}

Although sarcopenia is common in older adults, it can also occur early in life [2]. The reported prevalence of sarcopenia varies greatly depending on the population studied and the definition criteria, from 1 to $29 \%$ in elderly community-dwelling populations in western countries [3] and 2 to $46 \%$ in Asia [4]. This disorder is associated with higher rates of falls, decreased function, high mortality rates, and increased probability of hospitalization $[5,6]$.

Inflammation is a major risk factor for several conditions in the elderly. Inflammatory cytokines lead to rapid

(c) The Author(s). 2020 Open Access This article is licensed under a Creative Commons Attribution 4.0 International License, which permits use, sharing, adaptation, distribution and reproduction in any medium or format, as long as you give appropriate credit to the original author(s) and the source, provide a link to the Creative Commons licence, and indicate if changes were made. The images or other third party material in this article are included in the article's Creative Commons licence, unless indicated otherwise in a credit line to the material. If material is not included in the article's Creative Commons licence and your intended use is not permitted by statutory regulation or exceeds the permitted use, you will need to obtain permission directly from the copyright holder. To view a copy of this licence, visit http://creativecommons.org/licenses/by/4.0/ The Creative Commons Public Domain Dedication waiver (http://creativecommons.org/publicdomain/zero/1.0/) applies to the data made available in this article, unless otherwise stated in a credit line to the data. 
muscle wasting, eventually stimulate protein catabolism and suppress muscle synthesis [7]. Dietary factors have been shown to contribute to inflammation [8]. High intakes of fruits and vegetables [9], and also specific nutrients such as fiber [10], omega-3 fatty acids [11], vitamin E [12], and vitamin C [13] were associated with lower concentrations of circulating inflammatory markers. To determine the inflammatory potential of the whole diet, Dietary Inflammation Index (DII) has been designed and validated against circulating levels of inflammation [14, 15]. This dietary index has linked with several cancers, metabolic diseases, and fractures [16-18]. Two longitudinal cohort studies have also shown that higher DII scores were associated with a higher incidence of frailty [19] and decreased appendicular lean mass [8]. Given the limited studies on the association of inflammatory potential of the diet with muscle health, along with the great differences in dietary intakes, lifestyle factors and body composition of people in the Middle East with those in Western countries [20-22], this cross-sectional study aimed to investigate the relationship between DII with sarcopenia in an Iranian population.

\section{Materials and methods \\ Participants}

This population-based cross-sectional study was performed from May to October 2011 in Tehran, Iran. The detailed report on the sampling method and data collection procedure has been published previously [23]. In total, 300 elderly people (150 men and 150 women) with aged $\geq 55$ years were enrolled by the use of cluster random sampling method in district 6 of Tehran. The head of each cluster was selected based on a ten-digit postal code. To ensure the homogeneity of our sample, individuals whose potential cause of sarcopenia were factors other than aging were not invited. Indeed, people who were susceptible to sarcopenia due to secondary causes [24], including those who were unable to move, subjects with artificial limbs or limb prostheses, or individuals with debilitating diseases that predispose the person to sarcopenia (e.g malignancy, organ failure) were not included in the study.

\section{Dietary intake assessment}

Usual dietary intakes of the study participants was assessed using a 117-item Food Frequency Questionnaire (FFQ); the validity and reliability of this questionnaire was reported in previous studies [23, 25]. The questionnaire consisted of a list of foods with a specific portion size. Participants were able to report their consumption frequency based on daily, weekly or monthly basis for each food item. The questionnaire was filled by a trained nutritionist through face-to-face interview. After completing the FFQ, the frequency of each food item was converted to grams per day considering the household measures of portion sizes. Daily energy and nutrients intake of each participant was calculated by using Nutritionist IV software with a modified food composition database based on the US Department of Agriculture.

\section{Construction of dietary inflammatory index}

The method of Shivappa et al. was used to compute the Dietary Inflammatory Index (DII) [14]. DII score was calculated using 29 dietary parameters because some parameters suggested in the original scoring method were not available in our dataset in Iran. Dietary parameters included: energy, carbohydrate, fat, protein, fiber, cholesterol, mono-unsaturated fatty acids (MUFAs), polyunsaturated fatty acids (PUFAs), saturated fats (SFAs), vitamin B12, pyridoxine (B6), folic acid, niacin (B3), riboflavin (B2), thiamin (B1), vitamin A, C, D, E, b-carotene, zinc, selenium, magnesium, iron, caffeine, pepper, onion, garlic and green/ black tea. Residual method was used to obtain the energyadjusted amounts for all nutrients [26]. Then, to get zscore, the "standard global mean" was subtracted from the quantity of food and it was divided by the "global standard deviation". Standard global means and SDs for each dietary item were obtained from Shivappa et al. [14]. To decrease skewness, this value was converted to a centered percentile score. Then, this score was multiplied by the effect score for each of the food items obtained from Shivappa et al. [14]. Finally, to compute a total DII score for each participant, we summed the DII score obtained from all individual dietary parameters. The most negative score implies the maximum anti-inflammatory diet, while the most positive score implies the maximum pro-inflammatory diet.

\section{Assessment of sarcopenia}

Based on the European Working Group on Sarcopenia (EWGSOP) definition [24], sarcopenia was determined by considering the combination of both low muscle mass and low muscle function (either strength or performance). The muscle mass was measured as the ratio of an individual's total lean mass of legs and arms (also named Appendicular Skeletal Muscle or ASM) [27] to their squared height (ASM/height ${ }^{2}$ ). ASM was calculated with a DXA scanner (Discovery W S/N 84430). Based on EWGSOP, low muscle mass was considered as the amount of muscle mass less than $5.45 \mathrm{~kg} / \mathrm{m}^{2}$ for women and $7.26 \mathrm{~kg} / \mathrm{m}^{2}$ for men [24].

A handgrip test was used to measure muscle strength. The handgrip test was assessed by a pneumatic instrument that is a squeeze bulb dynamometer (c7489-02 Rolyan) calibrated in pound per square inch (psi). The handgrip strength (maximum voluntary contractions) was calculated three times for each right and left hand with a 30-s rest between measurements. Then, the average of these three measurements for each hand was calculated. Finally, the average number was obtained based 
on the sum of mean of both hands and this was considered as muscle strength. Sex and age-specific cutoff points recommended by Merkies et.al was then used to identify low muscle strength [28]. To measure muscle performance, a 4-Meter walk gait speed test was applied [24]. Participants who had gait speeds less than $0.8 \mathrm{~m} / \mathrm{s}$ were recognized as low muscle performance [24].

\section{Assessment of other variables}

Information about general characteristics of participant including age, sex, socio-economic status, medical history, medication use, smoking habits, and alcohol consumption were collected by a pre-tested questionnaire. The physical activity level in this study was examined by a trained interviewer using the short form of the International Physical Activity Questionnaire (IPAQ), its validity has previously been examined [25]. Measures of physical activity for each participant was expressed as metabolic equivalent-hour per week (MET-h/week) based on IPAQ's guideline [29]. Weight was measured using a digital scale while participants were minimally clothed. Height was measured by a wall tape meter in standing position without shoes. Waist circumference was measured in the middle of the lower rib margin and iliac crest while participants were stand up and normally breathe. Weight $(\mathrm{kg})$ divided by height squared $\left(\mathrm{m}^{2}\right)$ was used to calculate body mass index (BMI).

\section{Statistical analysis}

Subjects were classified according to the tertiles of DII score. General characteristics of study participants across tertiles of DII score were compared using Chi-square for categorical variables and ANOVA for continuous variables. Age- sex-, and energy-adjusted dietary intakes of participants were computed using General Linear Model and compared using ANCOVA across tertile categories of DII score. Multivariable logistic regression was conducted to find the relationship between inflammatory potential of the diet and odds of sarcopenia. In these analyses, several confounders were controlled. First, the association was adjusted for age (continuous), sex (male/female) and energy intake $(\mathrm{kcal} / \mathrm{d})$. Then, further controlling was done for physical activity (MET-h/wk), smoking (yes/no), alcohol consumption (yes/no), medication use (statin, corticosteroid, estrogen, testosterone), and positive history of chronic disease means subjects that affected by one of the following diseases: asthma, arthritis, myocardial infarction, cerebrovascular accident, and diabetes. In all these analyses, the bottom tertile was considered as the reference category and the odds ratio for sarcopenia in other categories was calculated. To evaluate the linear trend across tertiles of the DII score, the tertile categories were considered as an ordinal variable in the models. All analyses were done using SPSS (version 26). $P$-values were considered significant at $<0.05$.

\section{Results}

General characteristics of study participants across tertile categories of DII score are provided in Table 1. Subjects in the highest tertile were more likely to be older $(P=0.02)$ and less likely to be female $(P=0.008)$ than

Table 1 Characteristics of study participants in tertile categories of Dll score ${ }^{a}$

\begin{tabular}{|c|c|c|c|c|}
\hline & \multicolumn{3}{|c|}{ Tertiles of DII score } & \multirow[t]{2}{*}{$P$-value ${ }^{b}$} \\
\hline & $\mathrm{T}_{1}(n=100)$ & $\mathrm{T}_{2}(n=100)$ & $T_{3}(n=100)$ & \\
\hline Range of DII & $<-0.88$ & $-0.88,-0.70$ & $>-0.71$ & \\
\hline DII & $-1.95 \pm 0.74$ & $-0.03 \pm 0.46$ & $1.98 \pm 0.78$ & $<0.001$ \\
\hline Age (y) & $66.55 \pm 7.47$ & $65.47 \pm 7.01$ & $68.37 \pm 8.31$ & 0.02 \\
\hline BMI $\left(\mathrm{kg} / \mathrm{m}^{2}\right)$ & $27.58 \pm 4.32$ & $26.91 \pm 3.64$ & $27.64 \pm 4.60$ & 0.39 \\
\hline Physical activity (MET-h/w) & $1481.55 \pm 1464.81$ & $1170.93 \pm 1098.09$ & $1231.10 \pm 1663.12$ & 0.26 \\
\hline Female (\%) & 62 & 51 & 40 & 0.008 \\
\hline Alcohol use (\%) & 9 & 17 & 14 & 0.243 \\
\hline Smoking (\%) & 7 & 14 & 17 & 0.093 \\
\hline Medical history ${ }^{c}$ & & & & 0.739 \\
\hline Yes (\%) & 15 & 18 & 19 & \\
\hline No (\%) & 85 & 82 & 81 & \\
\hline \multicolumn{5}{|l|}{ Drug history } \\
\hline Sexual hormone use (\%) & 6 & 1 & 2 & 0.090 \\
\hline Statin use (\%) & 43 & 32 & 35 & 0.248 \\
\hline Corticosteroid use (\%) & 2 & 3 & 3 & 0.879 \\
\hline
\end{tabular}

${ }^{a}$ All values are mean $\pm S D$, unless indicated; ${ }^{b}$ ANOVA for continuous variables and Chi-squared test for categorical variables

c Medical history Yes means patients with one of the following diseases: asthma, arthritis, myocardial infarction, cerebrovascular accident, diabetes DII: Dietary Inflammatory index 
those in the lowest tertile of the DII score. The DII range was -3.98 to 4.29 , and participants in top tertile of DII had higher DII score indicating more proinflammatory diet than those in the bottom $(P<0.001)$. There was no other significant difference between the lowest and highest tertiles of DII in terms of mean BMI and distribution of participants in terms of alcohol use, smoking, medication use and disease history.

Table 2 presents age-, gender- and energy-adjusted dietary intakes of study participants across tertile categories of DII. Individuals in the highest tertile of DII had higher intakes of SFA, and lower intake of protein, iron, magnesium, zinc, thiamine, riboflavin, niacin, vitamin B6, b-carotene, vitamin A, vitamin C, dietary fiber, folate, pepper, onion, and garlic compared with those in the lowest tertile (all $P$-values were $<0.05$ ).

The prevalence of sarcopenia and its components across tertile categories of DII are shown in Table 3. Prevalence of low muscle mass and sarcopenia were significantly higher among subjects in the top tertile of DII than those in the bottom tertile $(P=0.041)$. There were no significant differences between means of muscle mass, handgrip strength and gait speed across tertile categories of DII after adjusted for age, sex, and energy.

Table 2 Dietary intakes of study participants by tertile categories of Dll score ${ }^{a}$

\begin{tabular}{|c|c|c|c|c|}
\hline & \multicolumn{3}{|c|}{ Tertiles of DII score } & \multirow[b]{2}{*}{$P$-value ${ }^{b}$} \\
\hline & $\mathrm{T}_{1}(n=100)$ & $\mathrm{T}_{2}(n=100)$ & $\mathrm{T}_{3}(n=100)$ & \\
\hline Range of DII & $<-0.88$ & $-0.88,-0.70$ & $>-0.71$ & \\
\hline Energy (kcal/d) & $2262 \pm 92.36$ & $2153 \pm 92.05$ & $2373 \pm 92.93$ & 0.25 \\
\hline \multicolumn{5}{|l|}{ Nutrients } \\
\hline Carbohydrates (g/d) & $368.32 \pm 5.51$ & $366.63 \pm 5.50$ & $363.12 \pm 5.56$ & 0.799 \\
\hline Proteins (g/d) & $89.55 \pm 1.78$ & $86.98 \pm 1.78$ & $81.66 \pm 1.80$ & 0.008 \\
\hline Total fats (g/d) & $58.15 \pm 1.89$ & $58.37 \pm 1.89$ & $61.33 \pm 1.91$ & 0.43 \\
\hline Cholesterol (mg/d) & $194.32 \pm 7.95$ & $195.94 \pm 7.94$ & $218.16 \pm 8.02$ & 0.068 \\
\hline $\mathrm{SFA}(\mathrm{g} / \mathrm{d})$ & $16.72 \pm 0.53$ & $17.51 \pm 0.53$ & $19.24 \pm 0.53$ & 0.004 \\
\hline MUFA (g/d) & $19.24 \pm 0.83$ & $18.88 \pm 0.83$ & $19.62 \pm 0.83$ & 0.824 \\
\hline PUFA (g/d) & $14.02 \pm 0.77$ & $14.15 \pm 0.77$ & $14.65 \pm 0.78$ & 0.838 \\
\hline $\mathrm{Fe}(\mathrm{mg} / \mathrm{d})$ & $21.53 \pm 0.32$ & $20.08 \pm 0.32$ & $18.48 \pm 0.32$ & $<0.001$ \\
\hline $\mathrm{Mg}(\mathrm{mg} / \mathrm{d})$ & $483.76 \pm 8.62$ & $450.09 \pm 8.61$ & $385.48 \pm 8.69$ & $<0.001$ \\
\hline $\mathrm{Zn}(\mathrm{mg} / \mathrm{d})$ & $12.65 \pm 0.28$ & $12.62 \pm 0.28$ & $11.64 \pm 0.28$ & 0.022 \\
\hline $\mathrm{Se}(\mathrm{mg} / \mathrm{d})$ & $0.097 \pm 0.003$ & $0.097 \pm 0.003$ & $0.098 \pm 0.004$ & 0.965 \\
\hline Thiamine (mg/d) & $2.31 \pm 0.04$ & $2.28 \pm 0.04$ & $2.09 \pm 0.04$ & 0.001 \\
\hline Riboflavin (mg/d) & $2.56 \pm 0.05$ & $2.39 \pm 0.05$ & $2.20 \pm 0.05$ & $<0.001$ \\
\hline Niacin (mg/d) & $21.91 \pm 0.37$ & $21.21 \pm 0.37$ & $20.16 \pm 0.37$ & 0.005 \\
\hline Vitamin B6 (mg/d) & $2.97 \pm 0.12$ & $2.71 \pm 0.12$ & $2.14 \pm 0.12$ & $<0.001$ \\
\hline b-carotene (mcg/d) & $3411.3 \pm 97.60$ & $2265.7 \pm 97.51$ & $1365.8 \pm 98.44$ & $<0.001$ \\
\hline Vitamin A (RE/d) & $2468.6 \pm 60.76$ & $1709.5 \pm 60.70$ & $1244.9 \pm 61.28$ & $<0.001$ \\
\hline Vitamin C (mg/d) & $355.26 \pm 8.51$ & $268.67 \pm 8.50$ & $201.12 \pm 8.58$ & $<0.001$ \\
\hline Vitamin E (mg/d) & $9.69 \pm 0.5$ & $9.17 \pm 0.5$ & $9.40 \pm 0.5$ & 0.760 \\
\hline Vitamin D (mcg/d) & $2.38 \pm 0.19$ & $1.85 \pm 0.19$ & $1.81 \pm 0.19$ & 0.069 \\
\hline Vitamin B12 (mcg/d) & $4.78 \pm 0.23$ & $4.65 \pm 0.23$ & $4.23 \pm 0.24$ & 0.240 \\
\hline Folate (mcg/d) & $617.99 \pm 9.65$ & $553.81 \pm 9.64$ & $458.84 \pm 9.73$ & $<0.001$ \\
\hline Dietary fiber (g/d) & $36.18 \pm 0.70$ & $29.53 \pm 0.70$ & $24.24 \pm 0.71$ & $<0.001$ \\
\hline Pepper & $25.59 \pm 2.69$ & $12.36 \pm 2.69$ & $7.17 \pm 2.71$ & $<0.001$ \\
\hline Onion & $42.22 \pm 3.17$ & $32.53 \pm 3.17$ & $24.81 \pm 3.20$ & 0.001 \\
\hline Garlic & $4.04 \pm 0.51$ & $2.09 \pm 0.51$ & $1.89 \pm 0.51$ & 0.006 \\
\hline Green/Black tea & $757.89 \pm 56.85$ & $824.39 \pm 56.80$ & $754.70 \pm 57.34$ & 0.578 \\
\hline Caffeine & $177.54 \pm 11.93$ & $182.46 \pm 11.89$ & $162.47 \pm 11.98$ & 0.477 \\
\hline
\end{tabular}

${ }^{a}$ All values are mean $\pm \mathrm{SE}$; energy intake is adjusted for age and sex, all other values are adjusted for age, sex and energy intake

${ }^{b}$ ANCOVA for all variables 
Table 3 Prevalence of sarcopenia and its components across tertile categories of DII score

\begin{tabular}{|c|c|c|c|c|}
\hline & \multicolumn{3}{|c|}{ Tertiles of DII diet score } & \multirow[t]{2}{*}{$P$-value ${ }^{a}$} \\
\hline & $T_{1}(n=100)$ & $\mathrm{T}_{2}(n=100)$ & $\mathrm{T}_{3}(n=100)$ & \\
\hline Range of DII & $<-0.88$ & $-0.88,-0.70$ & $>-0.71$ & \\
\hline \multicolumn{5}{|c|}{ Prevalence of components of sarcopenia } \\
\hline Low muscle mass (\%) ${ }^{b}$ & 33 & 35 & 49 & 0.041 \\
\hline Abnormal hand grip strength (\%) ${ }^{c}$ & 38 & 28 & 30 & 0.276 \\
\hline Abnormal gait speed $(\mathrm{m} / \mathrm{s})(\%)^{d}$ & 34 & 44 & 44 & 0.251 \\
\hline Sarcopenia (\%) & 14 & 13 & 27 & 0.016 \\
\hline \multicolumn{5}{|l|}{ Means of components } \\
\hline \multicolumn{5}{|l|}{ Muscle mass [ASM/h2] (kg) } \\
\hline Crude & $6.52 \pm 1.02$ & $6.61 \pm 1.00$ & $6.68 \pm 0.94$ & 0.526 \\
\hline Model $^{1}$ & $6.65 \pm 0.08$ & $6.59 \pm 0.08$ & $6.58 \pm 0.08$ & 0.814 \\
\hline \multicolumn{5}{|l|}{ Hand grip strength (psi) } \\
\hline Crude & $10.31 \pm 3.04$ & $11.60 \pm 3.88$ & $11.21 \pm 3.65$ & 0.031 \\
\hline Model $^{1}$ & $10.84 \pm 0.23$ & $11.42 \pm 0.23$ & $10.87 \pm 0.23$ & 0.145 \\
\hline \multicolumn{5}{|l|}{ Gait speed (m/s) } \\
\hline Crude & $0.86 \pm 0.20$ & $0.84 \pm 0.23$ & $0.82 \pm 0.23$ & 0.609 \\
\hline Model $^{1}$ & $0.87 \pm 0.02$ & $0.83 \pm 0.02$ & $0.82 \pm 0.02$ & 0.327 \\
\hline
\end{tabular}

Findings from multivariable logistic regression analysis indicating the association between inflammatory potential of diet and odds of sarcopenia are presented in Table 4. In the crude model, it was found that subjects in the highest tertile of DII had a higher likelihood of sarcopenia (OR: 2.27; 95\% CI: 1.10-4.65) compared with those in the lowest tertile. When the analysis was controlled for age, sex, and energy intake, participants with the greatest DII score had higher odds of sarcopenia (OR: 2.01; 95\% CI: 0.96-4.19) than those with the lowest DII, however, this was not statistically significant. Further adjustments for other covariates revealed that subjects in the top tertile of DII were 2.18 times (95\% CI: 1.01-4.74) more likely to have sarcopenia than those in the bottom tertile.

Furthermore, when the analyses were conducted for components of sarcopenia, we found that subjects in the top tertile of DII had a higher odds of low muscle mass (OR: 1.96; 95\% CI: 1.10-3.46) compared with those in the bottom tertile; however, this association disappeared when potential confounders were taken into account (OR: 1.38; 95\% CI: 0.72-2.63). There was no significant association between DII and abnormal hand grip strength as well as abnormal gait speed after controlling for covariates.

\section{Discussion}

Our findings indicated that increased DII score, indicating a more pro-inflammatory diet which is related to greater serum inflammatory markers, was associated with an increased odd of sarcopenia in older adults. This association remained significant even after adjustment for potential confounders. However, no significant association was seen between DII and components of sarcopenia including low muscle mass, abnormal handgrip strength, and abnormal gait speed. To our knowledge, this is the first study investigating the association between the inflammatory potential of the diet and sarcopenia.

In the current study population, total DII score ranged from -3.98 to 4.29 . We also found a positive association between higher DII scores and odds of sarcopenia. Although some studies had reported the relationship between DII and muscle mass and muscle strength, data on sarcopenia are limited. In a longitudinal cohort study in the US adults, DII was investigated in relation to frailty (defined as containing two of these criteria: weight loss, inability to rise from a chair 5 times, and poor energy). They reported that the range of DII in their participants was -5.65 to +3.70 and subjects in top quartile of DII score had a higher incidence of frailty in men [19]. Findings from a study on 1344 postmenopausal Korean women aged 50 years or older, revealed that the DII score range was -6.37 to +6.55 , and no significant association was found between higher DII score (higher versus lower than - 0.07) and osteosarcopenic conditions after adjustment for potential confounders [30]. Despite a positive association with sarcopenia, we failed to find 
Table 4 Multivariable-adjusted odds ratios $(95 \% \mathrm{Cls})$ for sarcopenia and its components across tertile categories of DII score

\begin{tabular}{|c|c|c|c|c|}
\hline & \multicolumn{3}{|c|}{ Tertiles of DII diet score } & \multirow{2}{*}{$\begin{array}{l}P \text { for } \\
\text { trend }\end{array}$} \\
\hline & $\overline{T_{1}}$ & $T_{2}$ & $T_{3}$ & \\
\hline Range of DII & $<-0.88$ & $-0.88,-0.70$ & $>-0.71$ & \\
\hline \multicolumn{5}{|l|}{ Sarcopenia } \\
\hline $\mathrm{N}$ & 100 & 100 & 100 & \\
\hline Crude & 1 & $0.91(0.40-2.06)$ & $2.27(1.10-4.65)$ & 0.018 \\
\hline Model 1 & 1 & $0.91(0.40-2.07)$ & $2.01(0.96-4.19)$ & 0.051 \\
\hline Model 2 & 1 & $1.01(0.43-2.39)$ & $2.18(1.01-4.74)$ & 0.035 \\
\hline \multicolumn{5}{|c|}{ Low muscle mass ${ }^{a}$} \\
\hline Crude & 1 & $1.09(0.60-1.96)$ & $1.96(1.10-3.46)$ & 0.021 \\
\hline Model 1 & 1 & $0.99(0.53-1.85)$ & $1.46(0.79-2.71)$ & 0.219 \\
\hline Model 2 & 1 & $0.98(0.51-1.88)$ & $1.38(0.72-2.63)$ & 0.312 \\
\hline \multicolumn{5}{|c|}{ Abnormal hand grip strength ${ }^{b}$} \\
\hline Crude & 1 & $0.63(0.35-1.15)$ & $0.69(0.38-1.25)$ & 0.226 \\
\hline Model 1 & 1 & $0.69(0.36-1.29)$ & $0.98(0.51-1.86)$ & 0.901 \\
\hline Model 2 & 1 & $0.66(0.34-1.29)$ & $0.97(0.49-1.89)$ & 0.902 \\
\hline \multicolumn{5}{|c|}{ Abnormal gait speed $(\mathrm{m} / \mathrm{s})^{c}$} \\
\hline Crude & 1 & $1.52(0.86-2.70)$ & $1.52(0.86-2.70)$ & 0.151 \\
\hline Model 1 & 1 & $1.88(1.02-3.46)$ & $1.78(0.96-3.29)$ & 0.064 \\
\hline Model 2 & 1 & $1.81(0.95-3.45)$ & $1.61(0.84-3.08)$ & 0.157 \\
\hline \multicolumn{5}{|c|}{$\begin{array}{l}\text { Model 1: Adjusted for age, sex and energy intake. Model 2: Further adjusted } \\
\text { for physical activity, smoking, alcohol consumption, medication use (statin, } \\
\text { corticosteroid, estrogen, testosterone), and positive history of disease (asthma, } \\
\text { arthritis, myocardial infarction, cerebrovascular accident, diabetes) } \\
\text { a Muscle mass lower than } 5.45(\mathrm{~kg} / \mathrm{m} 2) \text { for women and } 7.26(\mathrm{~kg} / \mathrm{m} 2) \text { for men } \\
\text { were considered low [24] } \\
\text { b Abnormal muscle strength was defined according previous study [28] } \\
\text { c Gait speeds lower than } 0.8 \mathrm{~m} / \mathrm{s} \text { were considered abnormal [24] }\end{array}$} \\
\hline
\end{tabular}

any significant association between DII and individual components of sarcopenia including low muscle mass, abnormal handgrip strength and abnormal gait speed. A prospective population-based study in Australia with 1099 men and women aged 50-79 years old revealed that the range of DII was -3.80 to +3.23 and each unit increase in DII score was not associated with a lower appendicular lean mass and handgrip strength after controlling for covariates [8]. It seems that additional information is required to shed light on the link between dietary inflammatory index and individual components of sarcopenia, given that there is a possible association between inflammation and sarcopenia, as indicated in a recent meta-analysis [7]. Low-grade chronic inflammation seems to predispose elderly people to muscle loss and dysfunction through affecting muscle proteolysis and myocyte apoptosis [31, 32]. Therefore, it is logical to expect finding an association between inflammatory potential of the diet and sarcopenia and its components.

Another finding of our study is that subjects in the third tertile of sarcopenia had significantly lower intakes of the anti-inflammatory components of DII including protein, iron, magnesium, zinc, thiamine, riboflavin, niacin, vitamin B6, b-carotene, vitamin A, vitamin C, folate, dietary fiber, pepper, onion, garlic than those in the first tertile. Moreover, participants in the top tertile had higher intakes of saturated fatty acids than those in the bottom tertile. This finding indicates that individuals with the greater adherence to a pro-inflammatory diet have lower intakes of fruit, vegetables, and protein and higher intakes of unhealthy fat. Based on the current dietary recommendations to live longer, high consumption of fruit and vegetables has been suggested [33-35]. A prospective cohort study among people aged $\geq 65$ years in Hong Kong showed that greater adherence to "vegetables-fruits" pattern and "snacksdrinks-milk products" pattern was associated with a lower odds of sarcopenia in men [5]. In the Newcastle 85+ cohort study, the investigators reported a positive association between a "traditional British" dietary pattern, characterized by high intakes of butter, red meat, gravy, and potato, and sarcopenia [36]. Existing evidence suggests that increased inflammation and production of pro-inflammatory cytokines (e.g CRP, IL-6, TNF-a) are associated with lower muscle mass, muscle strength, and physical performance [8]. The plausible mechanism might be the role of inflammatory cytokines in declining anabolic factors such as insulin and insulin-like growth factor-1 (IGF-1) through which they can down-regulate muscle protein synthesis [37]. Therefore, high intake of dietary factors with antiinflammatory properties may have beneficial effects on sarcopenia.

This study has several strengths. Several potential confounders were controlled for in the current analysis. Furthermore, a validated FFQ was used for the assessment of dietary intakes. The main limitation of our study is its cross-sectional design, which prohibits determining a causal relationship. Another limitation is the misclassification of study participants and measurement error due to the use of the FFQ. To reduce the effect of these errors, energy-adjusted DII was used in our study. Because of the limited data on some components of DII such as n-3 and n-6 fatty acids, eugenol, thyme/oregano, saffron, turmeric, trans fat, ginger, rosemary, and flavonoids, we did not include them in the DII calculation that may have caused an underestimation of the association; however, Shivapa et al. [14] showed that including at least 28 dietary parameters for calculating DII did not reduce the predictive ability of DII. Although the association between DII and inflammatory factors has been validated in previous studies [14], no information on serum inflammatory markers were available in the current study to examine its accuracy in the current population. In addition, it must be kept in mind that the current scoring of DII, as suggested by Shivappa et al. [14], was constructed based on available literature on diet and inflammation before 2010; however, a large body of 
evidence on the link between dietary factors and inflammation came out since that time. Therefore, it seems that a new updated scoring method, which take the current literature into account, must be developed. Lastly, the study was conducted on a small sample from the limited area where the DEXA device was located, because of financial limitations and inadequate access to the DEXA device in Tehran (maximum 300 cases). Accordingly, the generalization of these results to the whole Iranian population should be done with caution. Also, it is important to note that the current study was conducted on older adults. Therefore, extrapolation of the present findings to other age groups should be done cautiously.

\section{Conclusion}

In conclusion, greater adherence to a diet with more pro-inflammatory properties was associated with increased odds of sarcopenia in older adults. These findings support the current dietary recommendations on reducing the consumption of unhealthy foods with proinflammatory properties to reduce the risk of several chronic conditions including sarcopenia. Further welldesigned studies are required to investigate and clarify these associations.

\section{Abbreviations}

DII: Dietary Inflammatory Index; OR: Odds Ratio; Cl: Confidence Interval; SD: Standard Deviation; FFQ: Food Frequency Questionnaire; IPAQ: International Physical Activity Questionnaire; MET-h/week: Metabolic Equivalents-hours per week; BMI: Body Mass Index; SFA: Saturated Fatty Acid; MUFAs: mono-unsaturated fatty acids; PUFAs: polyunsaturated fatty acids; EWGSOP: European Working Group on Sarcopenia; ASM: Appendicular Skeletal Muscle; PSI: Pound per Square Inch

\section{Acknowledgments}

We wish to thank all individuals who kindly participated in our study.

\section{Authors' contributions}

$\mathrm{AB}, \mathrm{SS}, \mathrm{RH}, \mathrm{RH}, \mathrm{ADM}$ and $\mathrm{AE}$ contributed to the conception, design, data collection, statistical analyses, data interpretation, manuscript drafting, approval of the final version of the manuscript and agreed for all aspects of the work.

\section{Funding}

The financial support for this study comes from the Tehran Endocrine and Metabolism Research Center and the Tehran University of Medical Science.

\section{Availability of data and materials}

The data are not publicly available.

\section{Ethics approval and consent to participate}

The study protocol was approved by the Tehran University of Medical Sciences ethics committee. Initially, the aims of the study were explained to the participants and then all participants were requested to complete a written informed consent before data collection.

\section{Consent for publication}

The data provided to the researchers did not include any personal information, and all participants were adults. Not applicable.

\section{Competing interests}

The authors declare that they have no conflicts of interests.

\section{Author details}

'Students' Scientific Research Center, Tehran University of Medical Sciences, Tehran, Iran. ${ }^{2}$ Department of Community Nutrition, School of Nutritional Sciences and Dietetics, Tehran University of Medical Sciences, P.O. Box 14155-6117, Tehran, Iran. ${ }^{3}$ Department of Geriatric Medicine, Ziaeian Hospital, Tehran University of Medical Sciences, Tehran, Iran. ${ }^{4}$ Chronic Diseases Research Center (CDRC), Endocrinology and Metabolism Population Sciences Institute, Tehran University of Medical Sciences, Tehran, Iran. ${ }^{5}$ Obesity and Eating Habits Research Center, Endocrinology and Metabolism Molecular -Cellular Sciences Institute, Tehran University of Medical Sciences, Tehran, Iran. ${ }^{6}$ Department of Community Nutrition, Isfahan University of Medical Sciences, Isfahan, Iran.

Received: 22 July 2020 Accepted: 20 November 2020

Published online: 28 November 2020

\section{References}

1. Chen L-K, Lee W-J, Peng L-N, Liu L-K, Arai H, Akishita M, for Sarcopenia AWG. Recent advances in sarcopenia research in Asia: 2016 update from the Asian Working Group for Sarcopenia. J Am Med Directors Assoc. 2016;17: 767 e761-767. e767.

2. Cruz-Jentoft AJ, Bahat G, Bauer J, Boirie Y, Bruyère O, Cederholm T, Cooper C, Landi F, Rolland Y, Sayer AA. Sarcopenia: revised European consensus on definition and diagnosis. Age Ageing. 2019;48:16-31.

3. Cruz-Jentoft AJ, Landi F, Schneider SM, Zúñiga C, Arai H, Boirie Y, Chen L-K, Fielding RA, Martin FC, Michel J-P. Prevalence of and interventions for sarcopenia in ageing adults: a systematic review. Report of the international sarcopenia initiative (EWGSOP and IWGS). Age Ageing. 2014;43:748-59.

4. Chen L-K, Liu L-K, Woo J, Assantachai P, Auyeung T-W, Bahyah KS, Chou MY, Chen L-Y, Hsu P-S, Krairit O. Sarcopenia in Asia: consensus report of the Asian working Group for Sarcopenia. J Am Med Dir Assoc. 2014;15:95-101.

5. Chan R, Leung J, Woo J. A prospective cohort study to examine the association between dietary patterns and sarcopenia in Chinese community-dwelling older people in Hong Kong. J Am Med Dir Assoc. 2016;17:336-42.

6. Beaudart C, Zaaria M, Pasleau F, Reginster J-Y, Bruyère O. Health outcomes of sarcopenia: a systematic review and meta-analysis. PLoS One. 2017;12: e0169548.

7. Bano G, Trevisan C, Carraro S, Solmi M, Luchini C, Stubbs B, Manzato E, Sergi $G$, Veronese N. Inflammation and sarcopenia: a systematic review and metaanalysis. Maturitas. 2017;96:10-5.

8. Cervo MM, Shivappa N, Hebert JR, Oddy WH, Winzenberg T, Balogun S, Wu F, Ebeling P, Aitken D, Jones G. Longitudinal associations between dietary inflammatory index and musculoskeletal health in community-dwelling older adults. Clin Nutr 2020;39:516-23.

9. Root MM, McGinn MC, Nieman DC, Henson DA, Heinz SA, Shanely RA, Knab $A M$, Jin F. Combined fruit and vegetable intake is correlated with improved inflammatory and oxidant status from a cross-sectional study in a community setting. Nutrients. 2012;4:29-41.

10. Ma Y, Griffith JA, Chasan-Taber L, Olendzki BC, Jackson E, Stanek EJ III, Li W, Pagoto SL, Hafner AR, Ockene IS. Association between dietary fiber and serum C-reactive protein. Am J Clin Nutr. 2006;83:760-6.

11. Ferrucci L, Cherubini A, Bandinelli S, Bartali B, Corsi A, Lauretani F, Martin A, Andres-Lacueva C, Senin U, Guralnik JM. Relationship of plasma polyunsaturated fatty acids to circulating inflammatory markers. J Clin Endocrinol Metab. 2006;91:439-46.

12. Murphy RT, Foley JB, Tome M-T, Mulvihill NT, Murphy A, McCarroll N, Crean $\mathrm{P}$, Walsh MJ. Vitamin E modulation of C-reactive protein in smokers with acute coronary syndromes. Free Radic Biol Med. 2004;36:959-65.

13. Wannamethee SG, Lowe GD, Rumley A, Bruckdorfer KR, Whincup PH. Associations of vitamin C status, fruit and vegetable intakes, and markers of inflammation and hemostasis. Am J Clin Nutr. 2006;83:567-74.

14. Shivappa N, Steck SE, Hurley TG, Hussey JR, Hébert JR. Designing and developing a literature-derived, population-based dietary inflammatory index. Public Health Nutr. 2014;17:1689-96.

15. Tabung FK, Steck SE, Zhang J, Ma Y, Liese AD, Agalliu I, Hingle M, Hou L, Hurley TG, Jiao L. Construct validation of the dietary inflammatory index among postmenopausal women. Ann Epidemiol. 2015;25:398-405.

16. Orchard T, Yildiz V, Steck SE, Hébert JR, Ma Y, Cauley JA, Li W, MossavarRahmani Y, Johnson KC, Sattari M. Dietary inflammatory index, bone mineral 
density, and risk of fracture in postmenopausal women: results from the women's health initiative. J Bone Miner Res. 2017;32:1136-46.

17. Tabung FK, Steck SE, Ma Y, Liese AD, Zhang J, Caan B, Hou L, Johnson KC, Mossavar-Rahmani Y, Shivappa N. The association between dietary inflammatory index and risk of colorectal cancer among postmenopausal women: results from the Women's health initiative. Cancer Causes Control. 2015;26:399-408

18. Wirth M, Burch J, Shivappa N, Violanti JM, Burchfiel CM, Fekedulegn D, Andrew ME, Hartley TA, Miller DB, Mnatsakanova A. Association of a dietary inflammatory index with inflammatory indices and the metabolic syndrome among police officers. J Occup Environ Med. 2014;56:986.

19. Shivappa N, Stubbs B, Hébert JR, Cesari M, Schofield P, Soysal P, Maggi S, Veronese $\mathrm{N}$. The relationship between the dietary inflammatory index and incident frailty: a longitudinal cohort study. J Am Med Dir Assoc. 2018;19: 77-82.

20. Rush EC, Freitas I, Plank LD. Body size, body composition and fat distribution: comparative analysis of European, Maori, Pacific Island and Asian Indian adults. Br J Nutr. 2009;102:632-41.

21. Lear S, James P, Ko G, Kumanyika S. Appropriateness of waist circumference and waist-to-hip ratio cutoffs for different ethnic groups. Eur I Clin Nutr. 2010:64:42-61

22. Esmaillzadeh A, Azadbakht L. Food intake patterns may explain the high prevalence of cardiovascular risk factors among Iranian women. J Nutr. 2008;138:1469-75.

23. Hashemi R, Heshmat R, Motlagh AD, Payab M, Esmaillzadeh A, Baigy F, Pasalar P, Siassi F. Sarcopenia and its determinants among Iranian elderly (SARIR): study protocol. J Diabetes Metab Disorders. 2012;11:23.

24. Cruz-Jentoft AJ, Baeyens JP, Bauer JM, Boirie Y, Cederholm T, Landi F, Martin FC, Michel J-P, Rolland Y, Schneider SM. Sarcopenia: European consensus on definition and diagnosisReport of the European working group on sarcopenia in older PeopleA. J. Cruz-Gentoft et al. Age Ageing. 2010;39:412-23.

25. Mirmiran P, Esfahani FH, Mehrabi Y, Hedayati M, Azizi F. Reliability and relative validity of an FFQ for nutrients in the Tehran lipid and glucose study. Public Health Nutr. 2010;13:654-62.

26. Willett W, Stampfer MJ. Total energy intake: implications for epidemiologic analyses. Am J Epidemiol. 1986;124:17-27.

27. Heymsfield SB, Smith R, Aulet M, Bensen B, Lichtman S, Wang J, Pierson R Jr. Appendicular skeletal muscle mass: measurement by dual-photon absorptiometry. Am J Clin Nutr. 1990;52:214-8.

28. Merkies I, Schmitz P, Samijn J, Meché FVD, Toyka K, Van Doorn P. Assessing grip strength in healthy individuals and patients with immune-mediated polyneuropathies. Muscle Nerve. 2000;23:1393-401.

29. Committee IR. Guidelines for data processing and analysis of the International Physical Activity Questionnaire (IPAQ)-short and long forms. 2005. http://www.ipaq.ki.se/.

30. Park S, Na W, Sohn C. Relationship between osteosarcopenic obesity and dietary inflammatory index in postmenopausal Korean women: 2009 to 2011 Korea National Health and nutrition examination surveys. J Clin Biochem Nutr. 2018;63:211-6.

31. Zembroń-Łacny A, Dziubek W, Rogowski Ł, Skorupka E, Dąbrowska G. Sarcopenia: monitoring, molecular mechanisms, and physical intervention. Physiol Res. 2014;63:683-91.

32. Wang J, Leung K-S, Chow SK-H, Cheung W-H. Inflammation and ageassociated skeletal muscle deterioration (sarcopaenia). J Orthop Translat. 2017:10:94-101.

33. Lichtenstein AH, Appel L, Brands M, Carnethon M, Daniels S, Franch HA, Franklin B, Kris-Etherton P, Harris WS, Howard B. Diet and lifestyle recommendations revision 2006: a scientific statement from the American Heart Association nutrition Committee. Circulation. 2006;114:82-96.

34. Health UDo, Services H. Dietary guidelines for Americans 2015-2020. New York, NY: Skyhorse Publishing Inc., 2017.

35. Aune D, Giovannucci E, Boffetta P, Fadnes LT, Keum N, Norat T, Greenwood DC, Riboli E, Vatten $L$, Tonstad S. Fruit and vegetable intake and the risk of cardiovascular disease, total cancer and all-cause mortality - a systematic review and dose-response meta-analysis of prospective studies. Int J Epidemiol. 2017:46:1029-56.

36. Granic A, Mendonça N, Sayer AA, Hill TR, Davies K, Siervo M, Mathers JC, Jagger C. Effects of dietary patterns and low protein intake on sarcopenia risk in the very old: the Newcastle 85+ study. Clin Nutr. 2020;39:166-73.

37. Granic A, Sayer AA, Robinson SM. Dietary patterns, skeletal muscle health, and sarcopenia in older adults. Nutrients. 2019;11:745.

\section{Publisher's Note}

Springer Nature remains neutral with regard to jurisdictional claims in published maps and institutional affiliations.
Ready to submit your research? Choose BMC and benefit from:

- fast, convenient online submission

- thorough peer review by experienced researchers in your field

- rapid publication on acceptance

- support for research data, including large and complex data types

- gold Open Access which fosters wider collaboration and increased citations

- maximum visibility for your research: over $100 \mathrm{M}$ website views per year

At BMC, research is always in progress.

Learn more biomedcentral.com/submissions 